\title{
ADVANCED SUPERVISION OF OIL WELLS BASED ON SOFT COMPUTING TECHNIQUES
}

\author{
Edgar Camargo ${ }^{1}$ and Jose Aguilar ${ }^{2}$ \\ ${ }^{1}$ PDVSA. Edificio El Menito, LSAI Lagunillas, Edo Zulia-Venezuela \\ ${ }^{2}$ Universidad de Los Andes, CEMISID, Mrida, 5101, Venezuela \\ Prometeo Researcher, Universidad Tcnica Particular de Loja, Ecuador
}

\begin{abstract}
In this work is presented a hybrid intelligent model of supervision based on Evolutionary Computation and Fuzzy Systems to improve the performance of the Oil Industry, which is used for Operational Diagnosis in petroleum wells based on the gas lift (GL) method. The model is composed by two parts: a Multilayer Fuzzy System to identify the operational scenarios in an oil well and a genetic algorithm to maximize the production of oil and minimize the flow of gas injection, based on the restrictions of the process and the operational cost of production.

Additionally, the first layers of the Multilayer Fuzzy System have specific tasks: the detection of operational failures, and the identification of the rate of gas that the well requires for production. In this way, our hybrid intelligent model implements supervision and control tasks.
\end{abstract}

\section{Introduction}

The wrong functioning of industrial systems can cause financial and human losses, undesired environmental impacts, among others. This fact is true for a multitude of industrial domains: aerospace industry, oil companies, etc. Many of these systems are highly associated to automation. The automatic control frees them of the human manual control, but it is not immunized against operational failures. Therefore, it is necessary to complement the industrial automation systems with potent and accurate supervision tools that allow indicating undesired or unpermitted performance states, as well as taking the proper actions in order to keep the system within the optimal performance states.

The utilization of Hybrid Intelligent Systems (HIS) on supervision tasks in production systems is becoming an area of great interest at industrial level [1], [3], [5], [10]. Particularly, the HIS have gained a large influence in the oil industry, because they allow approaching the problem of handling the complexity of the hydrocarbon production systems [1], [2]. In special, the use of computational intelligence techniques represent an attractive alternative to deal with highly varying, complex, and confusing problems [7], [9].

So, in this work is proposed a HIS for supervising and optimizing oil production processes. The HIS is composed by a Multilayer Fuzzy Classifier System (MFCS) to detect faults, operational scenarios, and the rate of gas that the well requires for production; and a Genetic Algorithm (GA) to optimize the production. The MFCS consists of multiple fuzzy systems hierarchically distributed, each one for each task, which have the advantage that the total number of rules of the knowledge base is smaller, and are simpler than a conventional fuzzy system. The GA defines a population of individu- 
als, each of them representing a possible solution to the oil production optimization problem.

The main goal of the MFCS is the identification of different operational scenarios in an oil well, to implement control tasks. The MFCS carried out other supervision tasks (to detect faults that affect the process or the equipment involved, in real time, in the production facilities at the level of well and reservoir; to determine the rate of gas that the well requires for production) and it is the input to the GA. The system is initially tested in wells requiring artificial lift by Gas (ALG).

The main goal of the GA is the optimization of two objectives: the maximization of the production of hydrocarbons and the minimization of the gas injection. The GA must solve a zone of negotiation among these criteria that allows finding the ideal production.

The work herein presented reveals field tests of the system used to opmice (HIS) wells, based o the importante usage of axial load and several other variables (gas lift flow, pressures, etc) with a special ingredient that has a tremendous impact in optimization: Artificial Intelligence and Automation. The mathematical principles are also presented to encourage the usage of axial load in multivariable mathematical model in order to complete a good optimization scheme for these wells. The benefits of operating a HIS system using a artificial lift by Gas (ALG), are substancial, as describe in this paper. The idea is to reduce downtime, workovers, improve system operating response time and equipment useful life, while optimizing the well production.

This paper is structured as follows: Theoretical aspects about Fuzzy Classifier Systems and the Production Process of wells are presented in Section 2. The design of our HIS is presented in Section 3, the experiments with our HIS are shown in Section 4. The paper ends with conclusions.

\section{Theoretical Framework}

\subsection{Industrial Automation}

The Integral Automation Pyramid, proposed by the International Standards Organization (ISO), is associated with the hierarchical structure of decision-making processes. The base of the pyramid corresponds to the level of measurement and control, followed by a second level of Supervision, a third level of optimization, and finally, a last level of Asset Management. These same levels of the automation pyramid can be associated in three levels: the Operational Level for the task of control and measurement, the Tactical Level of Supervision and optimization tasks, and finally, the Strategic level.

Our HIS correspond to the Tactical Level, because it allows us to incorporate the following qualities to a system: autonomy in the decision making process; adaptation for the possibility of learning from the occurrence of events on the industrial system under supervision; self-diagnosing and self-organizing capacities anticipating the effect of the supervision tasks. Additionally, the HIS is distributed at process level, in a way that the decisions are carried out locally, thus minimizing the response times of the supervision tasks.

This approach extends the classical approach of the Data Control and Acquisition Systems (SCADA) that limit them to supervision and control tasks. This new approach is based on a selfregulation process in the wells, from the information they handle (status-actions), which allows them to anticipate situations, have a proactive behavior, without losing the global vision of the business.

Our HIS is oriented towards the provision of intelligence to the well by giving it onsite selfdiagnosing characteristics, in order to determine the production method with better performance and financial profitability. Wells with these characteristics have been called in the literature as "conscious wells", meaning by this term a well that, based on its profitability, regulates its production. For that, the well must have capabilities of self-diagnose, control of its damages, supervision of the behavior of its subsoil/surface infrastructure, etc. [7]. So, this allows providing intelligence to the production process through onsite self-supervision and self-optimization.

One of the most notable advantages of our approach is the architectural change towards a distributed intelligence model oriented to the field and the subsoil, eliminating the tactical level with the incorporation of the supervision and optimization tasks at the operational level (Figure 1). 


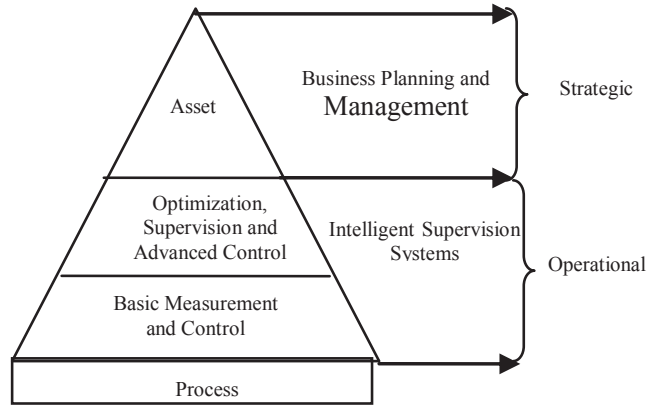

Figure 1. Pyramidal Automation Model based on our HIS

\subsection{Fuzzy Classifier System}

A Fuzzy Classifier System (FCS) is a system whose rules are based on the theory of Fuzzy Logic (FL), which includes the same elements of a Classifier Systems (CS), but working in a fuzzy framework [3], [4]. In this way, the activation of a rule is achieved when in the "antecedent" some values of fuzzy variables from the environment are activated. In standard fuzzy systems, for a problem with $\mathrm{n}$ input variables described by $\mathrm{m}$ linguistic labels, the maximum possible number of rules in the fuzzy system is $\mathrm{mn}$. This exponential growth causes, in practice, that for a high number of variables the number of rules is so large that the interpretability of the system becomes impossible. This problem is not exclusive to fuzzy systems, and is known as the problem of dimensionality [4].

One way of reducing the number of rules and thus increase the interpretability, is to decompose the fuzzy system into simple modules, this is called multilayer fuzzy systems (MFS) [5]. A MFCS consists of a number of fuzzy systems hierarchically distributed, which have the advantage that the total number of rules of the knowledge base is smaller, and are simpler than a conventional fuzzy system. There are numerous design proposals of such systems [5]. The more traditional type of MFS is one in which each module is a complete fuzzy system (FS) relates to a reduced set of variables, which can be input variables of the global system or internal variables generated as outputs of other modules [5]. There are other approaches, for example someone identify common set of rules and define common modules for them [7], or those in which each level corresponds to an increase in granularity of the variables [6]. Our work uses the first approach, which can be seen in Figure 2 [10].

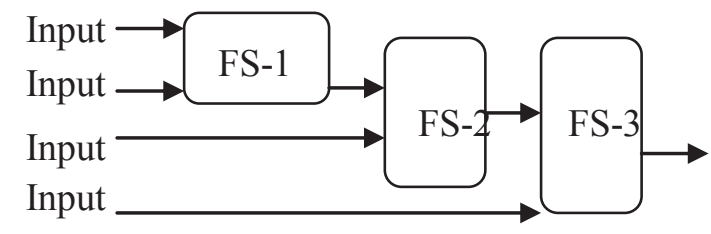

Figure 2. Classic Model of a Multilayer Fuzzy System

\subsection{Production Process of Wells by the Gas Lift Method}

The Gas Lift method consists of gas injecting at an established pressure at the lower part of the well pipe's fluid column, at different depths, with the purpose of decreasing its weight, thus helping the reservoir fluids rise from the bottom of the well to the surface. That way, in the wells exploited by the Gas Lift method the gas is continuously injected into the well in order to mix with the fluids of the well and reduce the density of the fluid column, thus decreases the difference in pressures between the bottom-hole and the surface.

The production curve of a well that produces by the gas injection method (see Figure 3) indicates that when the Gas Lift Flow increases (GLF, expressed "mpcdg" thousands of gas cubic feet days), the production rate (Qprod, expressed "BNPD" Daily Production Net Barrels) also increases, until reaching its highest value (Stable Region), such that additional increases in the injection will cause a decrease in the production (Unstable Region) [1], [2].

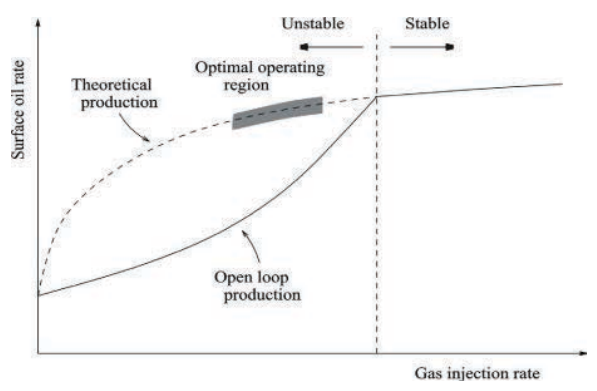

Figure 3. Artificial Gas Lift well behaviors model

The well's production curve is obtained by the characterization of the well using mass and energy balance techniques [1], [3]. The mechanical completion installed at the bottom and surface of the well allows the characterization of the physical 
properties of the fluid (Gravity of the oil, water cut, Bottom-hole pressure, Gas-liquid ratio). It is necessary because the oil production behavior in the wells injected with gas depends of variables, both of the reservoir and of the mechanical design (valves, production pipes, among others) [1]. The implantation of this ALG method needs an instrumentation and control arrangement. For that, the measurement and control of the following variables are required: Gas Lift Flow $\left(Q_{i n j}\right)$, Production Rate $\left(Q_{p r o d}\right)$, Gas Lift Pressure (Glp), Gas Lift Pressure Differential $($ Gldp $)$, Casing Pressure $\left(P_{g, i n j}\right)$, Production Tubing Pressure $\left(P_{t h p}\right)$ and Bottom Pressure $\left(P_{w f}\right)$.

So, a simple gas lift model is proposed [1]: the oil and gas "Inflow" of the reservoir is modeled with the use of the productivity index (oil volume that the reservoir can provide) and the existing relation between the production rate $\left(Q_{\text {prod }}\right)$ and the differential between the reservoir pressure $\left(P_{w s}\right)$ and the flowing pressure at the bottom of the well $\left(P_{w f}\right)$. Eq. (1) is used, which determines the capacity of contribution of the oil reservoir. This equation represents an instant of such capacity of contribution of the well of the reservoir, in a given time of its productivity life. It is normal for such capacity decreases through the time, due to the reduction of permeability of the well surroundings and the increase of viscosity of the oil. This equation is considered as the energy offered, or fluid affluence curve, that the reservoir yields to the well $\left(P_{w f} v s\right.$ $Q_{\text {prod }) \text {. }}$

$P_{w f}=P_{w s} *\left[\left(1,266-\frac{1,25 * Q_{\text {prod }}}{Q_{o}}\right)^{0,5}-0,125\right]$

Where $Q_{o}$ represents a base production rate, which is determined through reservoir core tests. As for the "outflow", gas is injected at a given depth to reduce the weight of the column and to reduce the bottom pressure of the well, allowing the establishment of a given production rate in which the capacity of fluid contribution from the reservoir equals the capacity of fluid extraction from the well. In this sense, in order to inject gas, it is assumed that the pressure at the level of the bottom injection valve located in the casing must be greater than the pressure in the space of the production pipe at the injection point $\left(P_{g, i n j\rangle} P_{T, i n j}\right)$, to ensure a displacement of the gas towards the production pipe. This is described by the following equation:

$Q_{i n j}= \begin{cases}c \sqrt{\rho_{g}\left(P_{g, i n j}-P_{T, i n j}\right)} & \left.\text { if } P_{g, i n j}\right\rangle P_{T, i n j} \\ 0 \quad & \text { else }\end{cases}$

Where,

$P_{g, i n j}=$ Pressure of Injection of Gas to the Valve

$P_{T, i n j}=$ Pressure of the Production Pipe at the Point of Injection

$\rho_{g}=$ Gas Density

$c=$ Constant related to the characteristics of the valve

$Q_{i n j}=$ Gas Injection Rate

For the model, the node at the gas injection valve is assumed in order to establish the capacity of production of the lifting system [2], [3]. Thus, the production of the system responds to an energy balance in the form of pressure between the capacity of energy contribution from the reservoir and the energy demand from the installation [2], which is expressed in the node as follow:

Node arrival pressure:

$$
\text { Pvalve }(\text { inflow })=P_{w s}-\Delta P_{y}
$$

Node output pressure:

$$
\text { Pvalve }(\text { outflow })=P_{\text {thp }}-\Delta P_{p}
$$

Where:

$\Delta P_{y}=P_{w s}-P_{w f}$ (Pressure Drop in the Reservoir)

$\Delta P_{p}=P_{t h p}-P_{T, i n j}$ (Pressure Drop in the Well) And now $\mathrm{Q}_{\text {iny }}$ is defined as:

$$
Q_{i n j}=c \sqrt{\rho_{g, i n j}\left(P_{g, i n j}-P_{t h p}+P_{w f}\right)}
$$

From equations (1), (2) and (3), the mathematical model that describes the behavior of a gas lift well is (4):

\section{Design of our HIS}

\subsection{Hybrid Intelligent Systems}

Our HIS is composed by a MFCS and a GA [10]. The MFCS consists of a number of fuzzy sys- 


$$
Q_{\text {prod }}=-\frac{Q_{O} *\left(\left(\frac{P_{t h p}+P_{g, i n j}-\left(\left(Q_{i n j} / c\right)^{2}\right) / \rho_{g}}{P_{w s}}+0,125\right)^{2}-1,266\right)}{1,25}
$$

tems hierarchically distributed, which allows identifying the different operational scenarios present in the oil production process. Additionally, the MFCS carries out other supervision tasks: detects faults that affect the process or the equipment involved; and determines the rate of gas that the well requires for production. Identified the operational scenario, the GA simulates the process of natural evolution to optimize the oil production. Every individual of the population represents a potential solution of the oil production problem. The evolution is guided by a strategy of selection of the individuals, with the intention of improving their "fitness", a measure based on the restrictions contextualized in the operational scenario determined by the MFCS. That means, the population of individuals will be specific to the operational scenario identified in the previous phase, so that the GA may optimize the production for that operational scenario.

\subsection{MFCS Design}

The proposed MFCS consists of 3 layers:

- The goal of the fist layer is to detect the faults that affect the process or the equipment involved. For that, the first layer determines the pressure drop in the production tubing. To calculate this drop are used the "bottom pressure" and "tubing pressure" (pressures that are present in the production tubing), which define the rules of the fuzzy system of the Figure 4.A. In this way, the first fuzzy system determines the intermediate linguistic variable " $P_{w f_{-} T h p}$ " (see Figure 4.A). So, the HIS starts with the input variables bottom pressure and production tubing pressure to obtain " $P_{w f-} T h p$ ", which is the pressure drop between those pressures. This characterization of the pressure drop in the production tubing of the well is important because it determines operational failures that may affect well production. So, the first layer defines a detection system of operational failures, based on rules which define the relationship Pwf vs Thp.

- The goal of the second layer is determined the rate of gas injection " $\mathrm{Q}_{i n j}$ " (see Figure 4.B), and with this value determine the operational stage of production of the well. With the operational stage of production of the well we can determine other operational faults due to an under-injection or over-injection of gas. In this sense, it consists of a set of rules that combine the pressure drop obtained in the first layer with the input variable "Casing Pressure", to get the gas injection rate. These rules use such variables because the rate of gas that is injected into the well to extract the oil to the surface depends on the pressure of the casing and the pressure drop in the tubing, according to [3], [5].

- Finally, the goal of the last layer is identify the operational scenario. For that, it determines the production rate (see Figure 4.C), and according to this value it can identify the operational scenario. In this case the set of rules are defined by the bottom pressure $\left(P_{w f}\right.$, fluid load capacity of the reservoir) and the gas injection rate $\left(Q_{i n j}\right.$, energy needed to extract the oil), because these variables determine the production rate according to [7], [8].

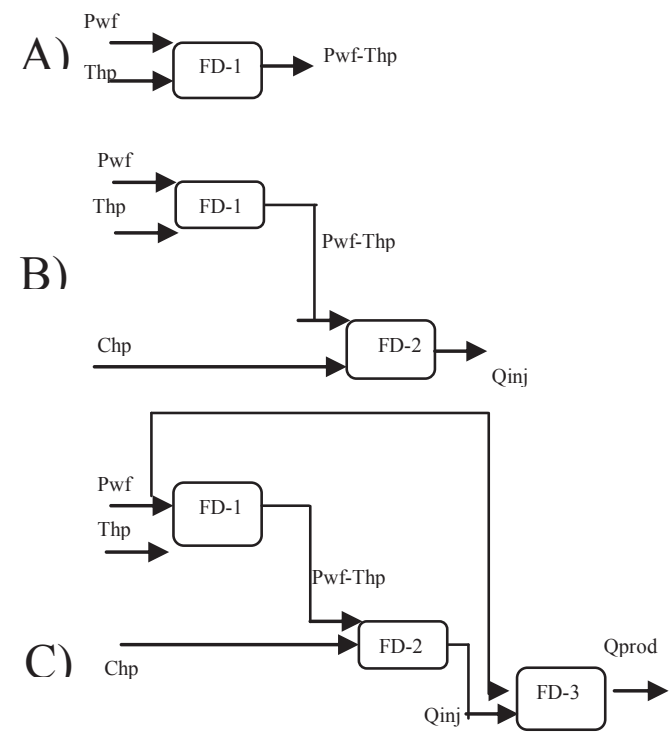

Figure 4. Our Multilayer Fuzzy Classifier System

With the output of the last level ( $Q_{\text {prod }}$, the production rate) the HIS determines the operational scenario of the well. Known the rate of production, the GA solves the problem of oil production optimization. 


\subsection{Optimization of the Production Pro- cess}

The optimization problem of ALG wells consists of increasing the production of oil and minimizing the flow of injected gas, based on three variables: Qprod, Cost and Qinj. This optimization problem is described by the objective function:

$f=($ PVPOil - Cost $\operatorname{ProdOil}) *$ Qprod - CostGas $*$ Qin $j$

Where,

PVPOil=Sell price of oil in terms of the daily barrel, $\$ / \mathrm{bl}$,

CostProductionOil=Production Cost,

CostGas=In \$/Mpcn.

And the restrictions of the process are: we assume that: $P_{w s}$ is a constant, due to the slow dynamics of the reservoir; and $P_{w f}$ is lower than the pressure of the reservoir, due to the fact that in a well the pressure of bottom is minor that the pressure of reservoir. Additionally, we establish the maximum production capacity that a reservoir can contribute as $Q_{\text {prod,max }}$, [3]. These restrictions are:

$$
\begin{aligned}
& P_{w s}=\text { Cons } \tan t . \\
& P_{w f}\left\langle P_{w s}\right. \\
& Q_{\text {prod }} \leq Q_{\text {prod,max }}
\end{aligned}
$$

Finally, the specific values of the variables $Q_{i n j}$, and $P_{w f}$ depend on the scenario identified in the previous phase. That is, the scenario identified determines the values of $Q_{i n y, \min }, Q_{i n j, \max }, P_{w f, \min }$, $P_{w f, \max }$. With these values, we define the next restrictions:

$$
\begin{aligned}
& Q_{i n j, \min \leq} \leq Q_{i n j} \leq Q_{i n j, \max } \\
& P_{w f, \text { min }} \leq P_{w f} \leq P_{w f, \text { max }}
\end{aligned}
$$

The structure of the individuals is composed by two fields that represent the variables Casing pressure $\left(P_{g, i n j}\right)$ and Tubing pressures $\left(P_{t h p}\right)$. These variables are used, because they are related to the gas behavior, and they can be manipulated at an operational level with an instrumentation arrangement. This is important, because such pressures can be adjusted in terms of the optimum values recommended by the GA, and thus achieve the best performances of the producing well (see equations (2), (3)
, and (4) in section II.B, which describe the model of gas injection defined in [1], [2]). In this way, the optimum value of production and injection is established according to the current operational scenario, using the equation (5), in a way that the set of values allowed to variables $P_{t h p}$ and $P_{g, i n j}$ depend on the operational scenario identified in the previous phase.

\section{Experiments}

The well characteristics where the system was implemented are the following: The completation of the producing vertical well is $3489 \mathrm{ft}$ and valves to $3184 \mathrm{ft}, 25$ API crude Gravity, 6\% water Cut. It receives gas lift from the gas Manifold located at $508,53 \mathrm{ft}$ far from it, and the Production Curve is shown in Figure 5.

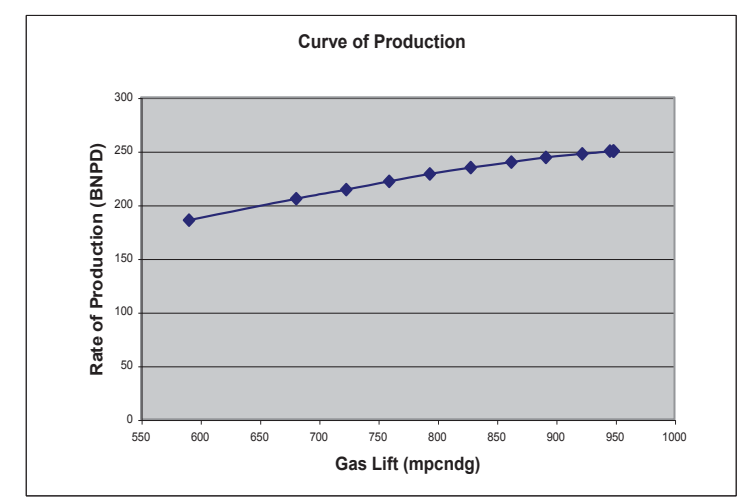

Figure 5. Experimental Production Curve by a Pressure of Reservoir to 2400 psi.

The behavior of the gas lift injection versus said production wells is the following: Well 1 operating at a gas injection rate mpcndg between 550 and 950 , and associated production well ranged between 190 and 250 BNPD. Well 2 operating at a gas injection rate mpcndg between 2000 and 8000 , and associated production well range between 4000 anb 6000 BNPD. These values were obtained at the flow station. In Table 1 and figure 6 are shown the current real production rate curve at the flow station (real 2400 psi curve), the curve which has been determined using the gas injection and the oil obtained at the station and evaluated in equation (4), and the theoretical curve. Using equation (4), families of curves can be created for different reservoir pressures (2600 psi, 2800 psi and 3000 psi), which show higher levels of production with respect to the 
pressure of theoretical field of 2400 psi, similar behavior as would occur in a real way. This is interesting because it allows us to determine the possible production curves presenting the well at different reservoir pressures. The model defined with the equations given above is specific to each LAG well, a new LAG well requires a similar procedure to determine its production curve. In the same way, we can calculate the production rate curves of the well 2 for reservoir pressures (4000 psi) (see figure 7, theoretical and real curves). it is important to note that the theoretical model follows the real curve, the value of average production is approximately $5753.53 \mathrm{~B} / \mathrm{D}$.

Table 1. Experimental Production Curve and Theoretical Curves for well 1

\begin{tabular}{|l|l|l|l|}
\hline $\begin{array}{l}Q_{i n j} \\
\text { Theoretical } \\
(2400 \text { psi) }\end{array}$ & $\begin{array}{l}Q_{\text {prod }} \\
\text { Theoretical } \\
(2400 \mathrm{psi})\end{array}$ & $\begin{array}{l}Q_{i n j} \\
\text { Real } \\
(2400 \\
\text { psi })\end{array}$ & $\begin{array}{l}Q_{\text {prod }} \\
\text { Real } \\
(2400 \\
\text { psi) }\end{array}$ \\
\hline 590,578 & 185,76 & 579,539 & 200,798 \\
\hline 680,824 & 205,55 & 625,619 & 210,215 \\
\hline 723,158 & 214,149 & 605,080 & 204,732 \\
\hline 759,345 & 221,87 & 598,212 & 203,567 \\
\hline 793,645 & 228,73 & 583,850 & 200,832 \\
\hline 828,213 & 234,72 & 598,803 & 203,888 \\
\hline 862,520 & 239,84 & 601,048 & 206,949 \\
\hline
\end{tabular}

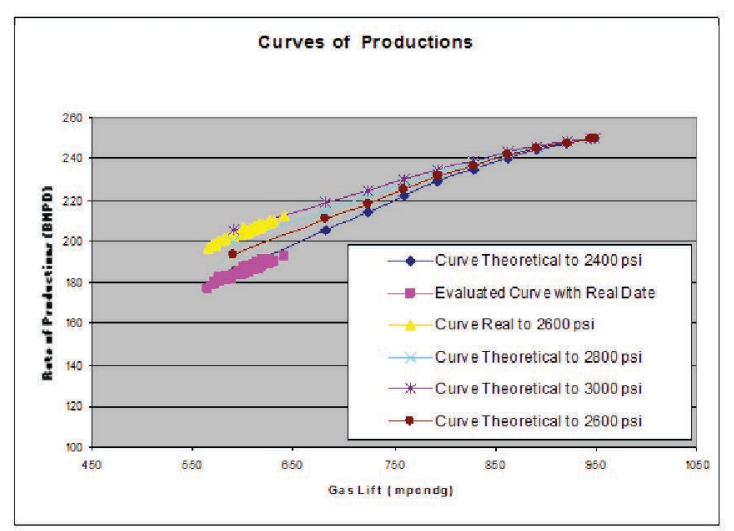

Figure 6. Experimental Production Curve and Theoretical Curves to different pressures of the reservoir for well 1.

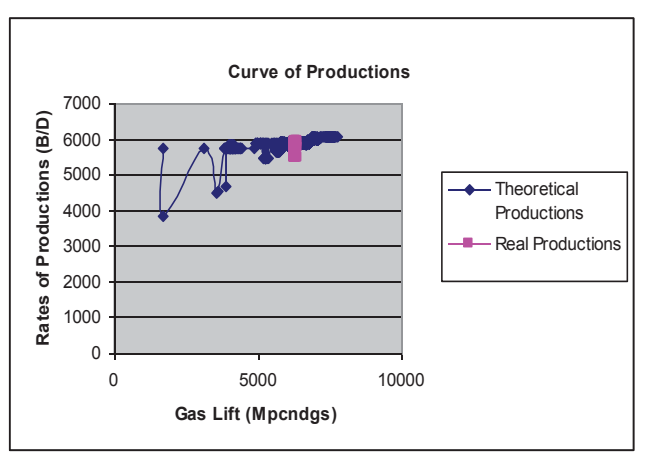

Figure 7. Experimental Production Curve for well 2.

Importantly, well 2 have different geometric characteristics and mechanicals the well 1 . It is a well of greater flow of oil, gas injected into the production tubing for three valves, and the completion of the producing vertical well is $12000 \mathrm{ft}$ with a pressure reservoir $4000 \mathrm{psi}$ and $4 \%$ water cut (see Table II). This well initially operated by natural flow, did not need mechanical or electrical energy to raise production. Due to the systematic deterioration of the reservoir pressure, has decayed its production, reason why now raises its production using the gas lift method, as is indicated in the following table.

Table 2. Experimental Production Curve and Theoretical Curves for well 2

\begin{tabular}{|l|l|l|l|}
\hline $\begin{array}{l}Q_{\text {inj }} \\
\begin{array}{l}\text { Theoretical } \\
(4000 \mathrm{psi})\end{array}\end{array}$ & $\begin{array}{l}Q_{\text {prod }} \\
\text { Theoretical } \\
(4000 \mathrm{psi})\end{array}$ & $\begin{array}{l}Q_{\text {inj }} \\
\text { Real } \\
(4000 \\
\text { psi })\end{array}$ & $\begin{array}{l}Q_{\text {prod }} \\
\text { Real } \\
(4000 \\
\text { psi })\end{array}$ \\
\hline 7590,571 & 5805,743 & 7624,532 & 5721,984 \\
\hline 7240,331 & 5731,243 & 7329,642 & 5843,218 \\
\hline 7943,491 & 6015,321 & 7862,218 & 5943,841 \\
\hline 7010,555 & 5642,921 & 7128,311 & 5541,921 \\
\hline
\end{tabular}

\subsection{MFCS: Identification of the Opera- tional Scenarios}

With the curve of the figures 6 and 7 and the historical data of the bottom and surface variables, we can characterize the input variables of the MFCS as follows (see Table III): 
Table 3. Membership Function for Input Variables for well 1

\begin{tabular}{|l|l|l|l|}
\hline & Chp (psi) & $P_{w f}(\mathrm{psi})$ & Thp(psi) \\
\hline Low & $1000-1120$ & $1-320$ & $150-200$ \\
\hline Medium & $1100-1220$ & $212-649$ & $190-260$ \\
\hline High & $1190-1320$ & $429-1093$ & $230-300$ \\
\hline
\end{tabular}

Table 4. Membership Function for Input Variables for well 2

\begin{tabular}{|l|l|l|l|}
\hline & Chp (psi) & $P_{w f}(\mathrm{psi})$ & Thp(psi) \\
\hline Low & $6140-6290$ & $1900-2150$ & $430-490$ \\
\hline Medium & $6200-6440$ & $2100-2300$ & $480-510$ \\
\hline High & $6430-6800$ & $2250-2400$ & $500-550$ \\
\hline
\end{tabular}

In the case of the output variable (Qprod) of the MCFS, its membership function is (Table V):

Table 5. Membership Function for output variables of wells

\begin{tabular}{|l|l|l|}
\hline $\begin{array}{l}\text { Operational } \\
\text { Scenarios }\end{array}$ & Well 1 & Well 2 \\
\hline Under-Injected & $400-600$ & $5500-6000$ \\
\hline Normal & $550-750$ & $6000-6500$ \\
\hline Over-Injected & $700-900$ & $6500-6800$ \\
\hline
\end{tabular}

The first layer characterizes the pressure drop in the production tubing of the well. This characterization is important because operational failures that may affect well production can be identified. So, to make that first detection of operational failures, we define a system of rules based on the relationship Pwf vs Thp, which gives their diagnosis. The Table VI shows the detection system of operational faults. It describes the different rules which define the operational diagnosis for different entries in our case studies: in each row, the first value of the first column is for the well 1 and the second for the well 2.

Regarding the second layer (FD-2), this allows us to identify the rate of gas that the well requires for production. With this value, we can determine the operational state of production of the well due to the gas injection rate derived from the pressure drop and the casing pressure. The operational state of production of the well defines new type operational faults due to the gas injection rate: under-injection and over-injection.
Table 6. Rules For Detection of Operational Failures FD-1

\begin{tabular}{|c|c|}
\hline $\begin{array}{l}\text { Pressure Drop: } \\
\text { Well } 1 \\
\text { Well } 2\end{array}$ & Operational Diagnosis \\
\hline $\begin{array}{l}3027,37 \\
6100,12 \\
\text { (High Drop) }\end{array}$ & $\begin{array}{l}\text { High hydrostatic pressure in } \\
\text { the tubing by: Low Flow of } \\
\text { Gas Injection. High Flow of } \\
\text { Gas Injection with presence of } \\
\text { High Water Cut. }\end{array}$ \\
\hline $\begin{array}{l}2890 \\
5580 \\
\text { (High Drop) }\end{array}$ & $\begin{array}{l}\text { High hydrostatic pressure in } \\
\text { the tubing by: Low Flow of } \\
\text { Gas Injection. High Flow of } \\
\text { Gas Injection with presence of } \\
\text { High Wate Cut. }\end{array}$ \\
\hline $\begin{array}{l}2870 \\
5020 \\
\text { (Medium } \\
\text { Drop) }\end{array}$ & $\begin{array}{l}\text { Medium hydrostatic pressure } \\
\text { in the tubing by: Low flow of } \\
\text { Gas Injection with presence of } \\
\text { High Water Cut and High Bot- } \\
\text { tom Pressure. High Flow of } \\
\text { Gas Injection with Leak Gas at } \\
\text { level of completation well. }\end{array}$ \\
\hline $\begin{array}{l}2229,7 \\
4995 \\
\text { (Medium } \\
\text { Drop) }\end{array}$ & $\begin{array}{l}\text { Medium hydrostatic pressure } \\
\text { in the tubing by: Low flow of } \\
\text { Gas Injection with High Wa- } \\
\text { ter Cut and Low Bottom Pres- } \\
\text { sure. High Flow Gas of In- } \\
\text { jection with Leak Gas at Level } \\
\text { Completation Well. }\end{array}$ \\
\hline $\begin{array}{l}2165 \\
4870 \\
\text { (Low Drop) }\end{array}$ & $\begin{array}{l}\text { Normal Flow of Gas and Pro- } \\
\text { duction }\end{array}$ \\
\hline
\end{tabular}

Table VII defines the rules of the detection system of these operational faults for our case studies: in each row, the first value is for the well 1 and the second for the well 2 . 
Table 7. Rules of detection of faults generated by the output of FD-2

\begin{tabular}{|l|l|l|l|}
\hline $\begin{array}{l}\text { Pressure } \\
\text { Drop: } \\
\text { Well 1 }\end{array}$ & Chp & $\begin{array}{l}\text { Operational } \\
\text { Scenario }\end{array}$ & $\begin{array}{l}\text { Qinj } \\
\text { MFCS: } \\
\text { Well 1 } \\
\text { Well 2 }\end{array}$ \\
\hline $\begin{array}{l}2164,78 \\
4870 \\
\text { (Low) }\end{array}$ & $\begin{array}{l}1190 \\
6430 \\
\text { (Medium) }\end{array}$ & UnderInj & $\begin{array}{l}517,8 \\
5250\end{array}$ \\
\hline $\begin{array}{l}2229,07 \\
4995\end{array}$ & 1320 & Normal & 666,67 \\
(Medium) & 6800 & & 6345 \\
\hline 2870,00 & 1250 & Normal & 735,23 \\
5020 & 6555 & & 6420 \\
(Medium) & (High) & & \\
\hline $\begin{array}{l}2890,00 \\
5580\end{array}$ & 1090 & OverInj & 776,17 \\
(High) & 6300 & & 6555 \\
\hline 3027,37 & 1020 & OverInj & 816,67 \\
6100,92 & 6150 & & 6740 \\
(High) & (Low) & & \\
\hline
\end{tabular}

Finally, the last layer (FD-3) determines the well production using the estimate rates of gas injection of the previous layer. With this value, the MFCS identifies the current operational scenario using the rules system defined in the Table VIII.

Table 8. Different Operational Scenarios determined by the MFCS

\begin{tabular}{|l|l|l|}
\hline Qprod MFCS: & Operational & Qinj MFCS: \\
Well 1 & Well 1 \\
Well 2 & & Well 2 \\
\hline 166,66 & UnderInj & 517,8 \\
5140 & & 5250 \\
\hline 200,38 & Normal & 666,67 \\
5529 & & 6345 \\
\hline 213,33 & Normal & 735,23 \\
5674 & & 6420 \\
\hline 243,09 & OverInj & 776,17 \\
5700 & & 6555 \\
\hline 252,09 & OverInj & 816,67 \\
6340 & & 7100 \\
\hline
\end{tabular}

\subsection{Optimization using GA}

The GA was applied for the operational scenario identified in the previous phase with MFCS, for our case study well 1 is analized for a Normal scenario and well 2 for an OverInj scenario. The optimization problem of LAG wells consists of increasing the oil production and minimizing the gas lift flow, based on the objective function and the operational restrictions described in section III.C (see equation (5)). In order to solve that problem, the GA used presents the following components:

Number of individuals: random, between 2 and 10 .

Number of generations: 25,

Objective function: equation (5), including its respective restrictions.

Crossover operator: single point cross with 0.7 probability.

Mutation operator: random with 0.03 probability.

The final population given by the GA for the normal operational scenario detected by the MFCS for the well 1 is shown in Table IX. An individual gives the values of $P_{t h p}$ and $P_{g, i n j}$, specified on a row of that table, which objective function is the value of Profits. That is, the optimum values for the normal operational scenario for the variables Tubing Pressure $\left(P_{t h p}\right)$ and Casing Pressure $\left(P_{g, i n j}\right)$ are shown in Table IX. These values are used in the models of gas injection for wells [1], [2] (see section II.B) and in the objective function (eq. 5), giving the results of $Q_{i n j}, Q_{\text {prod }}$ and Profits shown in the same Table IX.

Table 9. Results Obtained

\begin{tabular}{|c|c|c|c|c|}
\hline$P_{\text {thp }}$ & $P_{g, \text { inj }}$ & $Q_{\text {inj }}$ & $Q_{\text {prod }}$ & Profits \\
\hline 170 & 1022 & 596,6 & 232 & 7093 \\
\hline 170,4 & 1109,8 & 619,1 & 230,2 & 7034 \\
\hline 172,5 & 1226,3 & 689,1 & 233,7 & 7133 \\
\hline
\end{tabular}

According to the results of the Table IX, the production system presents an optimum behavior at a gas injection rate of about 596,6 mpcndg, with an associated production of $232,06 \mathrm{~b} / \mathrm{d}$, a casing pressure of $1022 \mathrm{psi}$ and production pipe of $170 \mathrm{psi}$. On the other hand, for a gas flow of 619,1 mpcndg its production rate is $230,21 \mathrm{~b} / \mathrm{d}$, generating a smaller profit and greater consumption of gas with respect to the case of 596,6 mpcndg. Regarding the gas flow of $689,1 \mathrm{mpcndg}$, a production of $233,71 \mathrm{~b} / \mathrm{d}$ is expected, higher than the one of 596,6 mpcndg $(1,64892 \mathrm{~b} / \mathrm{d})$, but more gas flow is required. In this 
case, the profit differential is $39 \$ / \mathrm{d}$, which indicates that this case could be interesting (more optimum) because it better combines the two costs.

In the case of the well 2 for an OverInj scenario, it presents an optimum behavior at a gas injection rate of about 5200 mpcndg with an associated production of $4934 \mathrm{~b} / \mathrm{d}$, a casing pressure of $6430 \mathrm{psi}$ and production pipe of $490 \mathrm{psi}$ (see Table X).

Table 10. Results Obtained

\begin{tabular}{|c|c|c|c|c|}
\hline$P_{\text {thp }}$ & $P_{g, \text { inj }}$ & $Q_{\text {inj }}$ & $Q_{\text {prod }}$ & Profits \\
\hline 490 & 6430 & 5200 & 4934 & 127752 \\
\hline
\end{tabular}

\section{Conclusion}

Our HIS uses MFCS and GA to define a control and supervision system for oil industrial production. The population of individuals in the GA correspond to the operational scenario identified with the MFCS, generating the optimum value of production and gas injection for this current operational scenario.

MFCS for the Analysis of Wells allows the analysis and classification of data from the well. It generates information from the reservoir variables (downhole pressure), head variables (casing pressure) and the gas flow. These variables are related to the gas injection process and its effect at the level of the reservoir. With such information it is more accurate the determination of the operational scenario of a well from its operating conditions, since in the same system the bottom and surface variables are integrated, different with respect to the current systems used in the industry, in which they only use surface variables. So, this will allow the self-diagnose of the well, monitor its damage, and care the performance of its infrastructure underground/surface. Specifically, our system allows estimating the rate of production and the gas injection rate, from which the well can improve its level of production to lower gas injection rate.

The production using AGL wells was optimized due to the integration of subsoil and surface information, which will allow minimizing costs and guaranteeing the best distribution of the gas injection maximizing the production of oil. The subsoilsurface integrated approach is innovative in the sense that it integrates the reservoir/wellhead infras- tructure behavior. This is carried out through an objective function, with the respective restrictions of the process, which allows contextualizing such objective function in the operational scenario and the reservoir conditions identified in the supervision scheme. The GA establishes the optimum production and the gas injection value for the identified operational scenario identified, from the relationship of the two costs of the productive process: reduce the production costs and optimize the gas injection.

The MFCS allows introducing a stepwise mechanism (in each layer) to detect/diagnose operational failures in each one of them, and thus throughout the completion of the Well. Each layer determines operational conditions from which we can establish the diagnosis of operational failures in the system. From this information, an online monitoring system could be developed at different levels of the well (for example, at the wellhead using the output of FD-1), with the aim of generating corrective actions based on the operational failure detected.

Our HIS needs a customization phase for the specific case study where it will be used. The production curve of the well and the historical data of the bottom and surface variables characterize the input variables of the MFCS. These input variables are specific for each case study (see tables I and II). Additionally, it is necessary define when a pressure is high, low, etc. for each case study (see tables III and IV), and its operational scenarios (see table V). Finally, the different layers (FDs) of the fuzzy system must be customized (tables VI, VII and VIII).

\section{Acknowledgment}

This work has been partially supported by Postgraduate Cooperation Program (PCP) between Venezuela and France entitled "Supervision and maintenance task in a shared organizational environment", No. 2010000307, between the University of Los Andes, Venezuela and LAAS-CNRS, France; and the Prometeo Project of the Ministry of Higher Education, Science, Technology and Innovation of the Republic of Ecuador.

\section{References}

[1] Edgar Camargo, Jose Aguilar, Addison Ros, Francklin Rivas, Joseph Aguilar-Martn, "Un mod- 
elo de Produccin de Pozos por Levantamiento Artificial utilizando Anlisis Nodal". Revista Ciencia e Ingeniera. Universidad de los Andes. Vol.30, pp. 23-28.N 1. 2009.

[2] Edgar Camargo, Jose Aguilar, Addison Ros, Francklin Rivas, Joseph Aguilar-Martn, "Nodal Analysis- based Design for Improving Gas Lift Wells Production". WSEAS Transactions on Informations Science \& Applications. Vol.5, No. 5, pp. 706-715. 2008.

[3] Edgar Camargo, Jose Aguilar, Addison Ros, Francklin Rivas, Joseph Aguilar-Martn, "A NeoFuzzy Approach for Bottom Parameters Estimation in Oil Wells", WSEAS Transactions on Systems and Control, Vol.4, No. 9, pp. 445-454, 2009.

[4] Robles O, Romn, R., "Un Modelo de Programacin No Lineal para la Planeacin de la Produccin de Gas y Petrleo". Paper SPE 112186, 2008 Intelligent Energy Conference, Amsterdam, Netherlands.

[5] Yang, D., Licheng J., Maoguo G. "Adaptive MultiObjective Optimization Based on Nondominated Solutions" Computational Intelligence, Volume 25, Number 2, 2009.
[6] Gong, M., L.C.Jiao., H.F.Du. "Multiobjective innmure algorithm with nondominated nieghbordbased selection" Evolutionary Computation, Volume 16, pp. 225-255, 2008.

[7] Shahab, D,M., "Recent Development in Application of Artificial Intelligence in Petroleum Engineering”. paper SPE 89033. Society of Petroleum Engineers. 2005.

[8] Popa A., Ramos R., Cover A., "Integration of Artificial Intelligence and Lean Sigma for LargeField Production Optimization: Application to Kern River Field”, Paper SPE 97247, pp. 34-45, 2005.

[9] Cordero, S., Moreno, F. "Una Herramienta de Induccin de Sistemas Difuso Jerrquicos". XV Congreso Espaol sobre Tecnologas y Lgica Difusa. Huelva (Spain), pp. 249-254. Feb. 2010.

[10] Edgar Camargo, Jos Aguilar, "Hybrid Intelligent Supervision Model of Oil Wells", Proceedings of the IEEE World Congress on Computational Intelligence (IEEE WCCI), Beijing, China, 2014. 